\title{
Functional Respiratory Changes in Patients with Chest Wall Deformities Secondary to Autologous Costal Cartilage Harvest for Auricle Reconstruction
}

Gutiérrez-Gómez C, ${ }^{1,2 *}$ Figueroa-Padilla J, ${ }^{3}$ Rosales-Chávez GA, ${ }^{4}$ Velasquillo-Martínez MC, ${ }^{2}$ Renán-León S, ${ }^{5}$ MartínezDelfín PS, ${ }^{6}$ González-Torres $\mathbf{M}^{7}$

${ }^{1}$ Departments of Plastic and Reconstructive surgery, Hospital General Dr. Manuel Gea González, Mexico City, Mexico

${ }^{2}$ Biotechnology Laboratory, National Burns Center, National Institute of Rehabilitation Luis Guillermo Ibarra Ibarra (INR LGII), Mexico

${ }^{3}$ National Cancer Institute, Mexico

${ }^{4}$ National Institute of Respiratory Diseases, Mexico

${ }^{5}$ Research, National Institute of Rehabilitation Luis Guillermo Ibarra Ibarra, Mexico

${ }^{6} \mathrm{Hospital} \mathrm{ABC}$, Mexico

${ }^{7}$ Conacyt-National Institute of Rehabilitation Luis Guillermo Ibarra Ibarra, Mexico

\begin{abstract}
Background: The gold standard for auricle reconstruction is currently performed with autologous costal cartilage. This process is done at about nine years of age, but it leads to thoracic deformity, reported in up to $70 \%$ of the patients using aComputed Tomography (CT) scanner.
\end{abstract}

Objective: The present study aims to determine if this deformity has functional implications for the patients.

Methods: 54 patients were clinically evaluated and subjected to spirometry at least one year after the surgery.

Results: Four cases had moderate pulmonary restriction, while seven had mild lung restriction. A total of $20.3 \%$ of the patients showed pulmonary restriction. The new results are particularly crucial for patients with preoperative (pre-op) ventilatory disease.

Conclusion: In patients with thoracic deformity diagnosed by clinic exploration, spirometric abnormalities occur in up to 20.3\%; when stratifying the risk by gender, the risk is only significant for women older than 15 years old.

Keywords: Auricle reconstruction, Respiratory function alterations, Thoracic deformity, Autologous auricle reconstruction sequelae, Microtia.

\section{Introduction}

Microtia (small ear) is a congenital deformity secondary to an alteration during embryonic development characterized by the partially or entirely underdeveloped pinna. ${ }^{1}$ The earliest reports of auricle reconstruction are described in the Sushruta Samhita, an ancient text, 900 BC. In 1930, Pierce reported a post-traumatic repair using costal cartilage. ${ }^{2}$ The previous findings paved the way for Tanzer, who pioneered modern costal cartilage reconstruction. ${ }^{3}$ Since then, the sixth to the tenth costal cartilages have been used in auricle reconstruction techniques. ${ }^{4-6}$ The pathophysiology of postoperative chest deformities can be explained in two ways, the first
Quick Response Code:

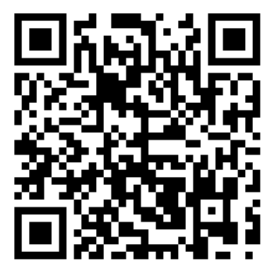

\footnotetext{
*Corresponding author: Claudia Gutiérrez-Gómez, Departments of Plastic and reconstructive surgery, Mexico, Puente de Piedra\#150 T2 C420 Col. Toriello Guerra Tlalpan, 14050 Mexico City, Mexico

Received: 07 June, 2021

Published: 28 June, 2021

Citation: Gutiérrez-Gómez C, Johnatan FP, Gustavo ARC, Cristina V, Saúl RL, et al. Functional Respiratory Changes in Patients with Chest Wall Deformities Secondary to Autologous Costal Cartilage Harvest for Auricle Reconstruction. Surg Int Open Acc J. 2021;1(1):1-5. DOI: 10.53902/SIOAJ.2021.01.000502
} 
one being the age at which cartilage resection is performed. Netscher found that the greatest thoracic cage growth occurs during the first four years of life, with a second peak between 12 and 18 years of age. ${ }^{7}$ This approach goes hand in hand with the mechanical factor proposed by Ohara, in which ribs with resected cartilage tend to curve inwards due to the effect of muscular force since they no longer have cartilaginous support. ${ }^{8}$ The second alternative shows that another important factor is the number of costal cartilages resected. Resection of the sixth costal cartilage produced deformity in $100 \%$ of the cases. However, the above procedure conducted in the seventh cartilage affected only $47.4 \%$ of the patients. Also, the reconstruction of the auricular framework is generally carried out before the age of 10 . Early reports recommend that it be carried out before the age range of 5-6 years. The recommendations have incorporated the psychosocial factors (i.e., school entry age) and the intervention at two years of age. ${ }^{9}$

Besides, the chest deformity resulting from cartilage resection can affect the patient's respiratory function. Spirometry has been used to evaluate respiratory function. This test shows the muscular effort of the thorax dynamically. It also indicates the interdependence of the alveoli with the retraction of the chest and lungs. The functional exploration of the respiratory system is an objective method to assess pulmonary function and quantify the degree of impairment caused by respiratory diseases. Forced spirometry is the most commonly performed functional test, and it can be carried out in patients as young as 5-6 years of age. The analysis of the forced expiratory volume in the first second (FEV1) allows us to classify the functional alteration quantitatively as mild, moderate, severe, and very severe. The analysis of FEV1, Forced Vital Capacity (FVC), and the ratio of both volumes (FEV1/FVC) allow to qualitatively classification pulmonary disorders in 3 different patterns: obstructive, restrictive, and mixed.

One of the first studies investigating the chondrogenic potential of the perichondrium discussed that the freed perichondrium could generate cartilage and that the new cartilage proliferates from the undersurface of the perichondrium. ${ }^{10}$ Tanzer followed up postoperative patients aged 6 to 19; they found that chest deformity occurred in $50 \%$ of the patients, associated with reconstruction carried out at an early age and mechanical factors that increase the thoracic cage's loss of stability. ${ }^{11}$ Ever since, efforts have been undertaken to reduce this complication by adjusting the surgical technique, such as sub-perichondria resection of the cartilage or suturing the periosteum after resection. However, the results have not been satisfactory. ${ }^{12,13}$ The study's main objective is to assess respiratory function in patients with chest deformity secondary to costal cartilage harvest for auricle reconstruction. Also, identify which respiratory alteration is found in these patients and a correlation between body mass index (BMI) and functional alterations.

\section{Methods}

\section{Study design}

This research is a descriptive, open, observational, prospective, and cross-sectional study. Patients diagnosed with microtia by the Plastic and Reconstructive Surgery® service at Dr. Manuel Gea González General Hospital have been included. Auricle reconstruction with rib cartilage was conducted by this service (at least a year before the study), with a clinical report of chest deformity and free acceptance of participation under informed consent and assent letters. In addition, patients with any congenital chest deformity, lung disease diagnosed before surgery, and patients who smoke and with a diagnosis of obesity (BMI>30) were excluded.

Postoperative patients with seventh to ninth costal cartilage harvest were analyzed in all cases. A clinical and spirometric control evaluation was performed at least one year after surgery. Sex, age, evident clinical deformity, and thoracic asymmetry were also documented.

The clinical evaluation was performed by the Plastic and Reconstructive Surgery service to detect asymmetry during respiratory movements. It included the "thumb test" (the physician places both hands around the patient's back from the anterior axillary line to an equidistant position at spine level). During deep inspiration, a lateral displacement of the thumbs occurs, noting the difference between both sides, if applicable. A direct medical history was taken to detect pre-existing conditions such as congenital anomalies, trauma, and previous reconstructive procedures not associated with auricle reconstruction that might occur with chest deformity. A pulmonologist performed the spirometric evaluation with a third-generation Henan Medical Spirobank II spirometer (Miami, Florida, USA). The evaluator was not informed of the results of the plastic surgeon's clinical evaluations carried out previously.

\section{Statistical analysis}

A bivariate analysis was conducted to compare means after checking the normality or non-normality of the distributions with Kolmogorov-Smirnov's Z. Student's t-test or Mann-Whitney U performed it. The comparison of proportions was conducted with a chi-square test. The intensity of the associations was calculated as relative risk with 95\% confidence intervals. The Mantel-Haenszel statistic was applied to compare risks by strata. Correlations were estimated with Pearson's r statistic or, when appropriate, Spearman's $\rho$. The receiver operating characteristic (ROC) curve was used to estimate the best cut-off point for a continuous variable that significantly differentiated the pulmonary restriction groups. The multivariate analysis for mean comparison was performed with a two-factor analysis of variance adjusted with analysis of covariance. The data were processed with the SPSS 17.0 package for Windows. In all statistical hypotheses, a $\mathrm{p}<0.05$ was assumed to reject the null hypotheses.

\section{Specialized terminology}

Clinical deformity: Defect observed in the thoracic contour evident through inspection by the evaluating physician (certified plastic and reconstructive surgeon).

Inspiratory asymmetry: Asymmetric expansion observed in the costal cage during the inspiration. 


\section{Results}

Fifty-four patients were evaluated; 26 (48.1\%) were female, and $28(51.9 \%)$ male. The average age was $15.5 \pm 6.1$ years old (ranged from 10 to 42). Because of the wide age margin, the distribution did not resemble the Gauss curve verified with the Kolmogorov-Smirnov test with $\mathrm{Z}=1.36(\mathrm{p}=0.04)$, as seen in Figure 1. The average body mass index was 20.8 \pm 3.3 (ranged at 13.4-28.4) with a normal distribution (Kolmogorov-Smirnov Z of 0.65, $\mathrm{p}=0.79$ ).

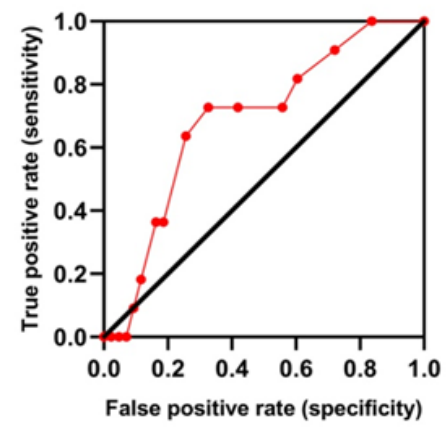

Figure 1: Interaction of pulmonary restriction, sex, and BMI.

The collapsed incidence of pulmonary restriction in ordinal to nominal as moderate/mild versus no restriction was $20.4 \%$ and associated with age. As shown in Table 1, the gender distribution is not significantly different ( $\mathrm{p}=0.63$ according to square Chi).The body mass index (BMI) neither was associated with pulmonary restriction ( $p=0.77$ according toStudent's t-test); instead, the average age in pulmonary restriction cases is significantly higher $(16.4 \pm 3.2$ years) when compared with those without restriction $(15.3 \pm 6.7$ years) with $\mathrm{p}=0.05$ according to no parametric $U$ of Mann.

Table 1: Comparative characteristics of patients with versus without pulmonary restriction with thoracic deformity secondary to costal cartilage resection for auricle reconstruction.

\begin{tabular}{|c|c|c|c|}
\hline \multirow{2}{*}{ Characteristics } & \multicolumn{2}{|c|}{ Pulmonary Restriction } & \multirow{2}{*}{} \\
\cline { 2 - 3 } & $\begin{array}{c}\text { Moderate/Mild } \\
\text { (n=11) }\end{array}$ & $\begin{array}{c}\text { Without } \\
\text { restriction } \\
\text { (n=43) }\end{array}$ & \multirow{2}{*}{ P } \\
\hline Gender & $6(54.5 \%)$ & $20(46.5 \%)$ & \multirow{2}{*}{0.63} \\
Female & $5(45.5 \%)$ & $23(53.5 \%)$ & \\
Male & $16.4 \pm 3.2$ & $15.3 \pm 6.7$ & 0.05 \\
\hline Age & $20.6 \pm 2.4$ & $20.8 \pm 3.5$ & 0.77 \\
\hline BMI & &
\end{tabular}

Figure 1 shows the ROC curve that identifies the best cut point of the age to separate the cases of pulmonary restriction. The best

Table 2: Correlation of BMI with sex and cases of pulmonary restriction.

Table 2: Correlation of BMI with sex and cases of pulmonary restriction.
\begin{tabular}{|c|c|c|c|c|c|}
\hline \multirow{2}{*}{ CRF-RP } & Sex & Media & Typical error & \multicolumn{2}{|c|}{ Confidence interval 95\% } \\
\cline { 3 - 6 } & Female & 20.86 & 1.31 & 18.22 & 17.14 \\
Upper limit \\
\hline \multirow{2}{*}{ Moderate/Mild } & Male & 20.01 & 1.43 & 18.24 & 23.49 \\
\cline { 2 - 6 } & Female & 19.68 & 0.72 & 20.65 & 21.11 \\
\cline { 2 - 6 } & Male & 21.99 & 0.67 & 23.32 \\
\hline
\end{tabular}

Conditional random field in pulmonary restriction (CRF-RP). cut point of the age was 15 years old (ROC curve area $0.687 \pm 0.08$ with $\mathrm{p}=0.05$ ).In figure 1 theROC curve of the susceptibility versus the specificity. The ties produce the diagonal segments. The hypothesis test variable result indicates that age has at least one tie-up between the real and real-state negative groups. The minor cut value is the hypothesis test value observed maximum plus one. The rest of the cut values are the media of two hypothesis test values observed ordered and consecutive. The age collapsed into two subgroups, namely, 16-42 and 10-15-year-old age group. The older age group showed more pulmonary restriction risk. A pulmonary restriction secondary to costal cartilage resection in $36.4 \%$ in the older than 15 years old group patients versus only $9.4 \%$ in the 10 15 years old group was noted.

The relative risk was 3.8(confidence interval (CI)=95\% of 1.1 13.0, $\mathrm{p}=0.01$ ). This result indicates 3.8 more pulmonary restriction risk in patients older than 15 years old than the 10-15-year-old age group. The risk stratification by age showed a significant difference only for females older than 15 years. This group also presented a pulmonary restriction relative risk of $6.8(\mathrm{p}=0.01)$. Conversely, the risk of the male stratum from the same age group was $2.3(\mathrm{p}=0.30)$ andwas not statistically significant. Furthermore, the value obtained from the Mantel-Haenszel chi-square test (4.1, p=0.04)confirmed that pulmonary restriction risks are statistically different between women and men. The two-way analysis of variance (ANOVA) with the covariance analysis carried out to adjust the BMI age is summarized in Table 2 and depicted in Figure 2. The covariable was 15.5 years old. As seen, the average BMI in the cases of pulmonary restriction between females and males is equal $(p=0.66)$. On the other hand, in the cases without pulmonary restriction, the BMI of the male group is significantly higher than the female group $(p=0.02)$. When comparing the groups with or without pulmonary restriction, the mean BMI value indicates no significant differences for either the women's ( $p=0.43$ ) or men's groups $(p=0.21)$.

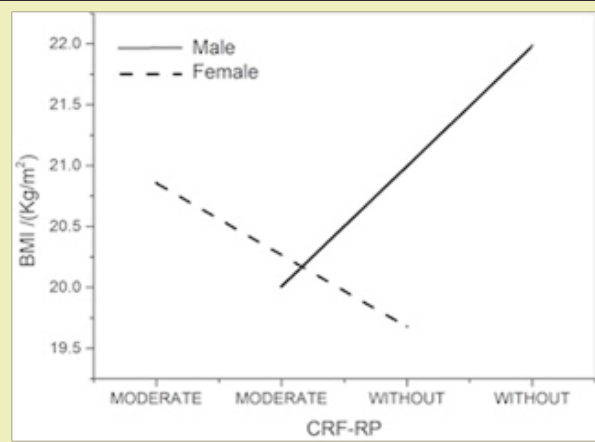

Figure 2: Shows the ROC curve that identifies the best cut point of the age to separate the cases of pulmonary restriction. 
The pulmonary interaction restrictionassigned to the variable *sex shows a $\mathrm{p}=0.15$, which tends to be significant. As seen in Figure 2, the female gender (as being with more risk) has a higher BMI media (20.86) in pulmonary restriction cases compared with no pulmonary restriction ones (19.68). By contrast, the male gender with pulmonary restriction revealed lower BMI media (20.01) than those without restriction (21.99). Nevertheless, none of the statistical hxpothesis tests are significant, confirming that the true associated factor to pulmonary restriction risk is exhibited for the females older than the 15-year-old age group.

\section{Discussion}

The thoracic deformity is one of the potential complications in treating patients undergoing costal cartilage harvesting for any purpose, either reconstructive or aesthetic. Earlier works have reported complications after harvesting costal cartilage with shortterm follow-up.

In patients with microtia, the thoracic deformity after taking the costal cartilage has been underestimated, and there are currently few reports in the current literature based on long-term follow-up. Thompson et al. ${ }^{9}$ published a retrospective study that reported chest deformity in 33\% of the cases where cartilage was taken at the ages of 2 to 3 compared to $8 \%$ when it was removed by age covering 6-12. ${ }^{9}$ Ohara confirmed chest deformity of $63.6 \%$ and $10 \%$ for cartilage removal before and after 10 -year-old patients, respectively. The above results demonstrated a correlation between age and the prevalence of chest deformity, where chest deformity was seen more in younger patients. ${ }^{8}$ Kawanabe and Nagata reported that the most critical factor in predicting postoperative chest deformity is not through the cartilage but via the technique with which it is removed. They suggested that preserving costal perichondrium and the subsequent adherence of the leftover fragments may reduce this complication. ${ }^{14,15}$

The respiratory alterations found in congenital chest deformities (i.e., scoliosis, rib fusion) are well known and have already been studied. ${ }^{16}$ However, there is no information regarding these alterations after costal-cartilage harvest to the best of our knowledge. For this reason, in our most recent study, we aimed to detected thoracic deformities secondary to costochondral harvesting using both imaging (CT scan) and clinical analysis. We found that taking three or more cartilages caused deformity in up to $70 \%$ of the patients, but only $30 \%$ were clinically evident. The anteroposterior thoracic diameter is the most affected section. In addition, the effect of the muscular insertion causes something that we call "pseudo-gynecomastia". We also found out that specific bone alterations are resulting from the cartilage resection pattern. The degrees of deformity helped us classify the patients to set a precedent and carry out future association measures. ${ }^{17}$ Two essential questions are: Would these deformities affect respiratory function, and could it be detected through spirometry? Should the diagnosis of alterations in ventilatory mechanics be considered beforehand in patients undergoing auricle reconstruction with rib cartilage harvest to consider the possibility of worsening ventilatory function?
The deformity is associated with the early age at which the reconstruction is performed. The mechanical factors that favor the lack of chest stability can also be associated with thoracic distortion. Previous works have suggested reducing this complication through changes in the technique with variable results. ${ }^{18}$ The purpose of spirometry is essentially limited to measuring the amount of air forcefully exhaled from fully inflated lungs, forced vital capacity (FVC), and the volume of air expelled in the first second of forced expiration, forced expiratory volume in the first second (FEV1). ${ }^{19}$ In our study, all patients with documented thoracic deformity were clinically evaluated; the latter based on the observations reported by the authors, where we found alterations in the anterior, posterior thoracic diameter and the muscular insertion. ${ }^{17}$ Spirometry is the simple expression of a complex process. Clinical studies on alterations in ventilatory function are basically for congenital conditions such as scoliosis and fusion of rib arches. ${ }^{16}$ However, there are no studies of ventilatory function for patients with deformity secondary to elective surgical procedures.

Thoracic deformity after taking costal cartilage for auricle reconstruction is a commonly underestimated complication. Our study demonstrates the presence of alterations in spirometry of patients with thoracic deformity secondary to costal cartilage, either clinically or radiologically diagnosed or presented. The incidence of pulmonary restriction was moderate and mild in 4 (7.4\%) and $7(13.0 \%)$, respectively.Alterations in pulmonary function were found in $20.4 \%$ of the cases. The pulmonary restriction - sex interaction yields a $\mathrm{p}=0.15$ that tends to be significant. The above results indicated that:

a) The female gender (being at higher risk) has a higher mean BMI (20.8) in the cases that presented pulmonary restriction compared to those that did not present it (19.6).

b) In men, the phenomenon is the other way around (that is the concept of interaction); those who presented pulmonary restriction had a lower BMI (20.0) than those without restriction (21.9).

However, none of the hypothesis tests are significant, which confirms that the true factor associated with the risk of pulmonary restriction is age $>15$ years associated with the female gender. No patient reported disability during daily activity. It is necessary to monitor growth in young patients to detect alterations since the muscular forces during growth exert changes on the bony structure of the thorax. Therefore, spirometry should be performed routinely in patients with thoracic deformity; it is a simple, inexpensive, and easy test to perform, and it must also be considered that the deformity may be accentuated during growth. In summary,our results suggest performing serial spirometry according to the patients' growth. This serial study would allow assessing a correlation between thoracic alterations and the growth of the rib cage.

\section{Acknowledgments}

Lic. Mariana Rodríguez Velasquillo for her invaluable help with the edition of the manuscript. Student Naomi Mariana Martínez Enriquez for the data analysis. 


\section{Funding}

None.

\section{Conflicts of Interest}

Author declares that there is no conflicto of interest.

\section{References}

1. Moore K, Persaud TVN. Embriología Clínica. $5^{\mathrm{a}}$ edition. Toronto: Ed. McGraw-Hill; 1995. p. 465-472.

2. Pierce G W. Reconstruction of the external ear. Surg Gynecol Obstet. 1930;395-401.

3. Tanzer R. Total reconstruction of the external ear. Plast Reconstr Surg. 1959;23:1-15.

4. Brent B. The acquired auricular deformity a systematic approach to its analysis and reconstruction. Plast Reconstr Surg. 1977;59:475-485.

5. Nagata S. Modification of the stages in total ear reconstruction of the auricle.Part 1. Grafting the three-dimensional costal cartilage framework for lobule-type microtia. Plast Reconstr Surg. 1994;93:221-230.

6. Latarjet M, Ruiz Liard A. Anatomía Humana. $3^{\mathrm{a}}$ edition. España: Panamericana; 1995. p. 969-975.

7. Netscher D, Peterson R. Normal and abnormal development of the extremities and trunk. Clin Plast Surg. 1990;17(1):13-21.

8. Ohara K, Nakamura K. Ohta E. Chest wall deformities and thoracic scoliosis alter costal cartilage graft harvesting. Plast Reconstr Surg. 1994;93:1030-1036

9. Thompson H, Kim T, Ein S. Residual problems in chest donor sites after microtia reconstruction: A long-term study. Plast Reconstr Surg. 1995;95:961-968
10. Skoog T, Ohlsen L, Sohn S. Perichondrial potential for cartilaginous regeneration. Scand J of Plast Reconstr Surg. 1972;6(2):123.

11. Tanzer R. Microtia: A long-term follow-up of 44 reconstructed auricles. Plast Reconstr Surg. 1978;61(2):161-166.

12. Laurie S, Kaban L, Mulliken J. Donor-site morbidity after harvesting rib and iliac bone. Plast Reconstr Surg. 1984;73(6):933-938.

13. Brent B. The correction of microtia with autogenous cartilage grafts: I. The classic deformity. Plast Reconstr Surg. 1980;66(1):1-12.

14. Skouteris CA, Sotereanos GC. Donor site morbidity following harvesting of autogenous rib grafts. J Oral Maxillofac Surg. 1989;47:808-812.

15. Kawanabe Y, Nagata S. A new method of costal cartilage harvest for total auricular reconstruction: Part I. Avoidance and prevention of intraoperative and postoperative complications and problems. Plast Reconstr Surg. 2006;117(6):2011-2018.

16. Campbell R, Smith M. The characteristics of thoracic insufficiency syndrome associated with fused ribs and congenital scoliosis. J Bone Joint Surg. 2003;85(3):399-408.

17. Figueroa J, Gutierrez C. Clinical and tomographic evaluation of thoracic deformities in unilateral auricular reconstruction with autologous costal cartilage: a useful tool for proper monitoring. I App Med Sci. 2013;2(4):1-9.

18. Uppal R, Sabbagh W, Chana J. Donor site morbidity after autologous costal cartilage harvest in ear reconstruction and approaches to reducing donor-site contour deformity. Plast Reconstr Surg. 2008;121:1949-1995.

19. American Thorax Society Medical Section of the American Lung Association. Standardization of Spirometry. 1994 Update. Am J Respir Crit Care Med. 1995;152(3):1107-1136. 